\title{
Optimization of a Loop-Mediated Isothermal Amplification Assay as a Point-of-Care Tool for the Detection of Wuchereria bancrofti in Human Blood in Tana River Delta, Kenya
}

\author{
Kinyatta Nancy $\mathbb{D}^{1},{ }^{1}$ Wambua Lillian, ${ }^{2}$ Mutahi Wilkinson, ${ }^{2}$ Mugasa Claire, ${ }^{3}$ Kamau Luna, ${ }^{1}$ \\ Wachira Dorcas, ${ }^{1}$ Githae Rosemary, ${ }^{1}$ Lusweti Japheth, ${ }^{1}$ Ichugu Christine, ${ }^{1}$ Waigi Emily, ${ }^{1}$ \\ and Kagai Jim ${ }^{1}$ \\ ${ }^{1}$ Kenya Medical Research Institute, Centre for Biotechnology Research and Development, P.O. Box 54840-00200, Nairobi, Kenya
${ }^{2}$ School of Biological Science, University of Nairobi, 30772-00100, Nairobi, Kenya
${ }^{3}$ College of Veterinary Medicine, Animal Resources and Biosecurity, Makerere University Kampala, 7062 Kampala, Uganda
}

Correspondence should be addressed to Kinyatta Nancy; nkinyatta@kemri.org

Received 23 November 2020; Revised 28 June 2021; Accepted 7 July 2021; Published 28 July 2021

Academic Editor: Bernard Marchand

Copyright (C) 2021 Kinyatta Nancy et al. This is an open access article distributed under the Creative Commons Attribution License, which permits unrestricted use, distribution, and reproduction in any medium, provided the original work is properly cited.

\begin{abstract}
Introduction. Accurate detection of filarial parasites in humans and vectors is essential for the implementation and evaluation of Global and National Programs to eliminate lymphatic filariasis. Immunological methods to detect infection are available; however, cross-reactivity issues have been reported in most of them. Nucleic acid-based molecular assays offer high levels of specificity and sensitivity and can be used to detect the infections. Methods. In this study, we evaluated loop-mediated isothermal amplification (LAMP) tests to amplify Wuchereria bancrofti DNA in patients' blood. The amplicons were tested by both $\mathrm{pH}$-sensitive dyes for enhanced visual detection and agarose gel electrophoresis. A closed-tube LAMP assay was also evaluated. Cohen's Kappa statistics was used for statistical analysis of the assays. 125 patients consented for blood sampling which were used for clinical analysis of LAMP assays with the PCR method used as the "gold standard." Results. The sensitivity of the evaluated Wuchereria bancrofti LAMP was $92.3 \%$, with a specificity of $97.3 \%$ and kappa statistics value of 0.84 , which is in a strong agreement. Conclusion. In this study, LAMP assays coupled with fluorescence dye detection have been found to be suitable for diagnosis and monitoring of Wuchereria bancrofti infections in the Kenyan population.
\end{abstract}

\section{Introduction}

1.1. Background Information and Literature Review. Lymphatic filariasis (LF) is a chronic parasitic disease of public health and socioeconomic significance in tropical and subtropical countries. It is caused by the filarial worms of Wuchereria bancrofti, Brugia malayi, or B. timori species. This debilitating mosquito-borne nematode infection had been earmarked for elimination by the year 2020 [1]. The disease is transmitted by five genera of mosquitoes, Culex, Aedes, Anopheles, Mansonia, and Ochlerotatus [2], making it the most common vector-transmitted parasitic infection after malaria [3,4]. It is the second leading cause of permanent and long-term disability worldwide after eye blindness
[5]. Acute and chronic morbidity resulting from lymphatic filariasis has affected 120 Million people living in 81 endemic countries with 1.34 billion people at risk of developing the infections [6]. In 2000, the Global Program to Eliminate LF (GPELF) was launched [7] and it was estimated that 5-6 rounds of mass drug administration (MDA) with ivermectin or diethylcarbamazine and albendazole were required to eliminate the disease [5]. In 2018, 893 million people in 49 countries were living in areas that required preventive chemotherapy to stop the spread of the infections [8].

Early and accurate diagnosis of Wuchereria bancrofti causing bancroftian filariasis is a key factor in the effort to eliminate filariasis [9]. The diagnosis has relied on the detection of microfilariae in blood specimen and in mosquitoes 
vectors [9]. Point-of-care diagnosis of lymphatic filariasis is largely based on microscopic examination of blood collected at night $(2200 \mathrm{pm}-2000 \mathrm{am})$ [10]. The immunochromatographic test (ICT) is a rapid format which detects specific circulating filarial antigen in bancroftian filariasis [11, 12]. Immunochromatographic test (ICT) is regarded by the WHO as the "gold standard" for diagnosis of lymphatic filariasis [11]. It has a number of limitations which include high cost and inconsistent availability and also detects antigen even after clearance of the worms on treatment [11]. Microfilariae antibody detection for brugian filariasis is available in clinical settings $[12,13]$. However, the antibody tests indicate exposure rather than active infection [14] and do not distinguish between bancroftian and brugian filariasis [15-17]. Following the implementation of the control programs, monitoring is necessary to determine the endpoint of treatment, with continued surveillance being required to identify areas of ongoing transmission or recrudescence $[11,16]$. These activities and overall management of MDA programs can be achieved efficiently by the use of accurate, sensitive, specific, and relatively cheap diagnostic tools suitable for point-of-care application.

Molecular-based amplification methods such as polymerase chain reaction (PCR) are proven technologies with high sensitivity and specificity [18-20] for the detection of microfilariae. These methods have not been widely used in remote settings because of the high cost and complexity of the procedures requiring trained personnel and sophisticated equipment [21]. Several PCR-based methods have been used to amplify DNA in blood from B. malayi- and B. timori- [22] and $W$. bancrofti-infected patients $[18,23,24]$. Molecular monitoring of insect vectors by PCR is also the preferred method for xenodiagnoses that has been used extensively for $W$. bancrofti $[25,26]$ and B. malayi $[27,28]$. An alternative to PCR are the isothermal amplification techniques which amplify DNA at a relatively constant temperature; thus, a simple water bath or a heat block can be used [29-33].

Loop-mediated isothermal amplification (LAMP) is a unique and novel amplification method with high specificity and sensitivity able to discriminate between single-nucleotide differences [29]. It is characterized by the use of six different primers specifically designed to recognize eight distinct regions on a target gene, with amplification only occurring if all primers bind and form a product [29]. In the past, LAMP has been successfully applied for the rapid detection of both DNA and RNA viruses such as the West Nile [34] and SARS viruses [30]. Parasitologists have adapted the LAMP approach for the detection of several parasitic diseases including the human parasites Entamoeba [35], Trypanosoma [36], Taenia [37], Plasmodium [38], and Cryptosporidium [39]. Other parasite detection assays include the animal parasites Theileria and Babesia [40, 41]. LAMP has also been developed for the identification of vector mosquitoes carrying Plasmodium and Dirofilaria immitis parasites [42]. Most of these studies have brought to light the many advantages of this method over the common PCR technique. In addition, LAMP results can readily be assessed by white precipitate formation from large amounts of pyrophosphate ions as a by-product generated during the reactions, and also, the results can be assessed by the naked eyes or by adding a fluorescence dye $[36,43]$. The applicability of LAMP to field surveys and hence as a diagnostic and mapping tool for filarial infections was first demonstrated by detecting Dirofilaria in wild-caught mosquitoes [44]. Later, Takagi et al. [32] found out that the LAMP method was able to detect $W$. bancrofti DNA in human blood and mosquito pools and a potential tool for field applications with more validation studies.

Due to limitations of most of the diagnostic methods highlighted, for a precise reveal of the distribution of filarial infections, novel diagnostic methods that are simple, rapid, sensitive, and reliable are required. In consideration of these points, this study was to evaluate and validate LAMP as a molecular isothermal amplification assay for diagnosis of Wuchereria bancrofti to aid in monitoring the prevalence of the disease in Kenyan endemic areas.

\section{Materials and Methods}

2.1. Study Site. Samples for this study were collected from Tana River Delta in Tana River County, Kenya (Figure 1, showing a map of the Tana River Delta). This region is one of the endemic areas considered to have high prevalence (22.2\%) [45] where MDA Programs started in 2011 [46]. Tana River Delta was curved off from Tana River district in 2007. It has 3 divisions Kipini, Garsen, and Tarasaa with an area of $16013 \mathrm{~km}^{2}$. Tana River County has a population of 250000 with 134000 in Tana River district according to the 2009 census [47]. The rainfall ranges between 220 and $900 \mathrm{~mm}$ per year, while the average temperature is $30^{\circ} \mathrm{C}$. The altitude ranges between 0 and $200 \mathrm{~m}$. The major ethnic groups are the Pokomo, who are subsistent famers and practice fishing, and Orma and Wardey who are predominantly nomadic.

2.2. Ethical Clearance. The permission to carry out this study was sought from the Kenya Medical Research Institute Scientific and Ethical Review Unit (SERU), protocol number SSC. 2802. Participants had been requested to consent (adults) or assent (children) to participate in the study by giving the blood sample to be used in this study.

2.3. LAMP Primer Designing. The primers used to perform the present assay were those previously identified by Takagi et al. [32]. The target was 18s rRNA species-specific regions (Ssp1), a highly repeat gene on the W. bancrofti complete sequence of accession number (AY297458), yielding 188 base pairs. The diagrammatic representation of sets of primers targeting Wuchereria bancrofti mitochondria DNA is as shown in Table 1.

2.4. LAMP Primer Specificity Testing. Before optimizing the LAMP assays, the specificity of the outer primers (F3 \& B3) was tested by conventional PCR. The expectations here were that primers specific for $W$. bancrofti detection would show amplification only on positive controls and with no amplification on negative controls. The positive controls used here were $W$. bancrofti commercial controls and a known positive specimen as earlier determined by conventional PCR with NV1 and NV2 Ssp1 repeat sequence primers. The positive 


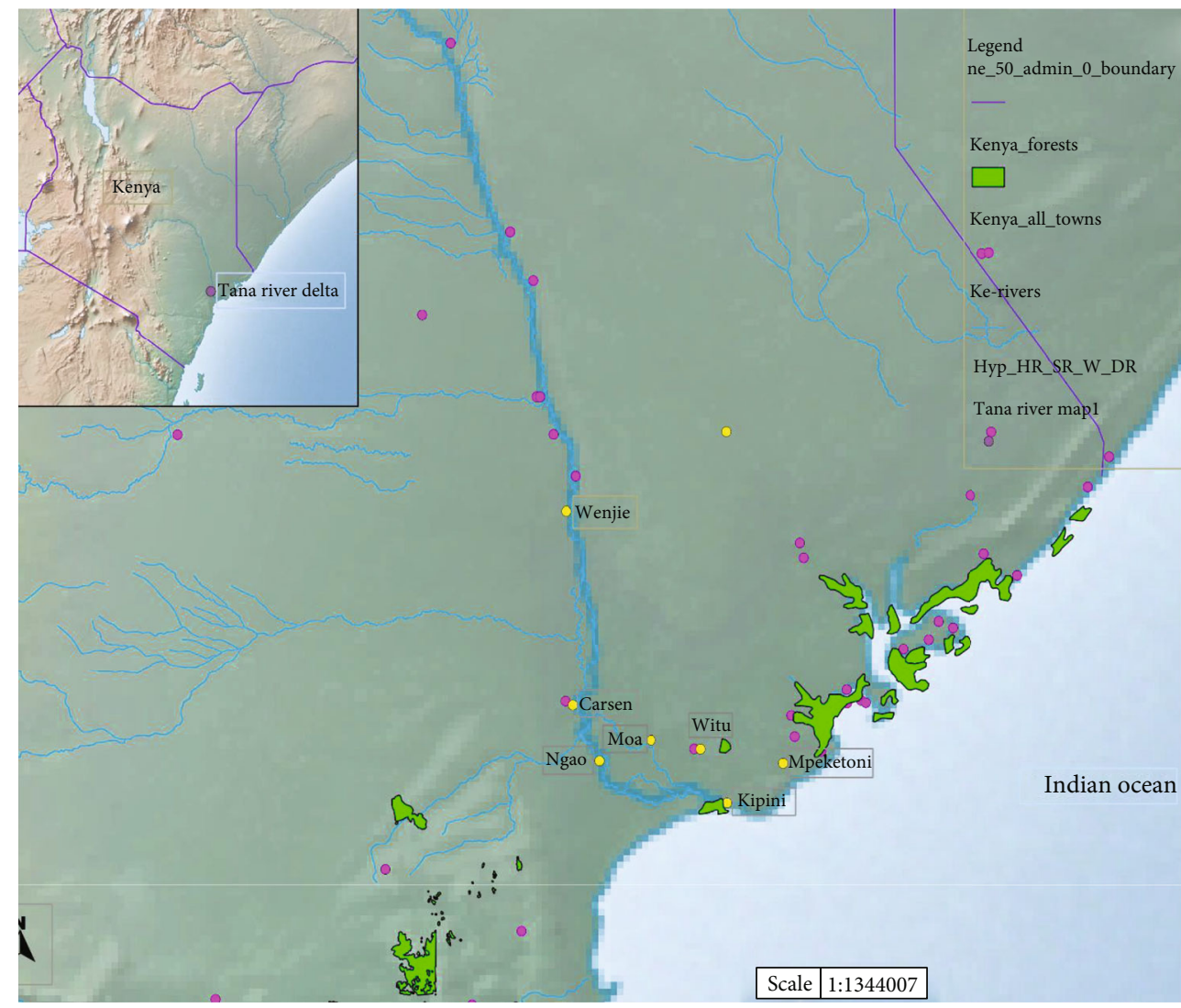

Figure 1: Map of Tana River Delta in Tana River County, Kenya. Source: map generated by Jacob Mueti.

TABLE 1: LAMP and PCR primer sets used for the detection of $W$. bancrofti in this study.

\begin{tabular}{|c|c|c|c|c|}
\hline Target repeat sequence & Amplification assay & Primer position & Sequence $\left(5^{\prime} \ldots \ldots \ldots \ldots \ldots \ldots 3^{\prime}\right)$ & Purpose \\
\hline \multirow{6}{*}{ Ssp 1} & \multirow{4}{*}{ LAMP primers } & $\mathrm{FIP} ; \mathrm{F} 1_{\mathrm{C}}+\mathrm{F} 2$ & $\begin{array}{l}\text { CGACTGTCTAATCCATTCAGAGTG- } \\
\text { TATCTGCCCATAGAAATAACTACG }\end{array}$ & Forward inner primer \\
\hline & & $\mathrm{BIP} ; \mathrm{B} 1_{\mathrm{C}}+\mathrm{B} 2$ & $\begin{array}{l}\text { TCTGTGCTGAATTTTTGTGGATTG- } \\
\text { CCAAACTAATTGTAAGCAGTCTT }\end{array}$ & Reverse inner primer \\
\hline & & F3 & TTTGATCATCTGGGAACGT & Forward outer primer \\
\hline & & B3 & AAGCACCTTAAATCTGTCAAT & Reverse outer primer \\
\hline & \multirow{2}{*}{ PCR primers } & NV-1 & $5^{\prime}$-CGTGATGGCATCAAAGTAGCG-3'; & Forward primer \\
\hline & & $\mathrm{NV}-2$ & $5^{\prime}$-СССТСАСТTACCATAAGACAAC-3'. & Reverse primer \\
\hline
\end{tabular}

controls had been sequenced earlier with accession numbers MK471341 and MK 471347 on the gene bank. The negative controls included blood specimen from the nonendemic area, blank Master Mix, and the PCR water as initially confirmed by PCR.

2.5. Sample Processing and Amplification. Blood samples were collected from consenting patients into well-labeled vacutainers. Samples were subjected to DNA extraction by alcohol precipitation method as described by Datta et al. [48] with minor modifications. The quantity and quality of the extracted DNA was determined by measuring A260 and the ratio of A260/A280 on a NanoDrop ND-1000 spectrophotometer (Thermo Scientific, USA). The DNA was amplified by LAMP and polymerase chain reaction assays for comparison. Detection of the amplicons was by agarose gel electrophoresis and 1:10 SYBR Green 1 dye or a florescence dye.

2.5.1. Polymerase Chain Reaction (PCR). The PCR was performed as originally reported by Nicolas and Plichart [18]. Two primers as identified by Zhong and colleagues [49] as $\mathrm{NV}-1$ and NV-2 were used. The PCR reaction mix contained $12 \mu \mathrm{l}$ of $10 \mathrm{x}$ Bioline buffer with $\mathrm{Mgcl} 2$ and dNTPs, $5 \mathrm{pmol} / \mu \mathrm{l}$ of NV1 and NV2 primers each, $5 \mu$ l genomic DNA template, and water to top up the reaction volume to $25 \mu \mathrm{l}$. The PCR reaction was run in a 96-well GeneAmp ${ }^{\circledR}$ PCR system 9700 with conditions consisting of a single step of $95^{\circ} \mathrm{C}$ for 5 minutes, 40 cycling step of $94^{\circ} \mathrm{C}$ for 30 seconds, $54^{\circ} \mathrm{C}$ for 45 seconds, $72^{\circ} \mathrm{C}$ for 30 seconds, and a final extension step of 
$72^{\circ} \mathrm{C}$ for 10 minutes. The PCR products were size fractioned on $2.0 \%$ agarose gel stained with Ethidium bromide. Agarose gel electrophoresis was run at $80 \mathrm{~V}$ for 60 minutes and bands visualized under UV light using a gel documentation system (EZ Imager, Bio-Rad, CA). Positive control and negative controls were included in every run to ensure specificity and validity of the results. The expected $W$. bancrofti size band was 188 base pairs and this was measured against a 100 base pair molecular weight marker.

2.5.2. LAMP Optimization. Optimization of the LAMP assay was done by varying the reaction temperatures and time, as well as varying the concentrations of LAMP primer sets of forward and backward outer primers (F3 and B3) and forward and backward inter primers (FIP and BIP). The final assay-optimized conditions were as follows: the final reaction mixture of $25 \mu \mathrm{l}$ contained primers ( $40 \mathrm{pmol}$ of FIP and BIP and 5 pmol of $\mathrm{F} 3$ and $\mathrm{B} 3$ outer primers), DNA polymerase, 8 units of Bst I large fragment (Meridian Bioscience ${ }^{\circledR}$ ), $1 \mathrm{mM}$ dNTPs, $0.8 \mathrm{M}$ betaine, and $1 \mathrm{x}$ reaction buffer (containing 20mMTris- $\mathrm{HCl}, \mathrm{pH} 8.8,10 \mathrm{mM} \mathrm{KCl}, 10 \mathrm{mM}$ (NH4) $2 \mathrm{SO} 4,8 \mathrm{mMMgS} 4$, and $1 \%$ Tween 20 ). The reaction was incubated at varying temperature ranging from $60^{\circ} \mathrm{C}$ to $65^{\circ} \mathrm{C}$ on a heat block for time between 30 minutes and 60 minutes. During the optimization process, DMSO $7.5 \%$ as per assays by Wang et al. [50] was added to reduce false-positive results due to primer dimers.

To make LAMP amplification more applicable in a field setup, the Commercial Isothermal Amplification kit was used from Eiken Chemical Co. (Tochigi, Japan). Direct detection of amplicons in a reaction tube was done by direct observation of the reaction with the unaided eye for the color change after addition of $1 \mu \mathrm{l}$ of $1: 10$ SYBR Green I dye (Invitrogen, Carlsbad, CA) to the amplicon. The DNA product was also visualized under ultraviolet light at $320 \mathrm{~nm}$ after electrophoresis on $2 \%$ standard agarose gel for 60 minutes at $80 \mathrm{~V}$ and then photographed for records.

2.5.3. Closed-Tube Detection: Lyophilized LAMP Reaction Buffers. To further assess LAMP application in a field setup, closed-tube detection to reduce aerosol pollutants and crosspollutant with SYBR Green 1 for detection was used. The reaction kit-Loopamp DNA amplification reagent-contains all essential reaction components (reaction buffers and dNTPs) in a dried form on the cap of each reaction tube. For the reaction, only the specific primers and the DNA template were added and incubated at appropriate conditions. The Commercial Isothermal Amplification kit was used from Eiken Chemical Co. (Tochigi, Japan). Direct detection of amplicons in a reaction tube was done by direct observation of the reaction with the unaided eye for turbidity in the presence of amplified Wuchereria bancrofti DNA and viewed under UV light for any color change in addition to $1 \mu \mathrm{l}$ of $1: 10$ SYBR Green I dye (Invitrogen, Carlsbad, CA).

2.6. Analytical LAMP Specificity Testing. The specificity of the LAMP assay was carried out using $W$. bancrofti-specific primers to amplify DNA of other related parasites including Brugia malayi [27], Schistosoma mansoni, Plasmodium falci-

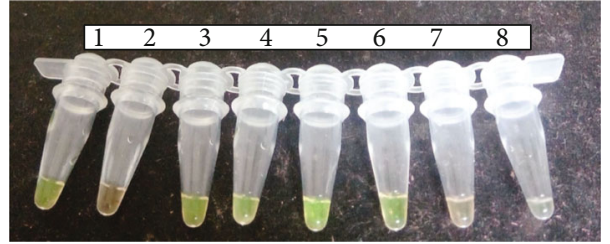

FIgURE 2: LAMP florescence dye detection (open tubes). Tube no. 1: $W$. bancrofti DNA-positive control; 2: negative control; 3, 4, 5, and 6: positive specimens; 7 and 8: negative specimens. Green color indicates that Wuchereria bancrofti DNA was present and was amplified (positive). Orange color means that there was no $W$. bancrofti DNA and hence no amplification that took place (negative).

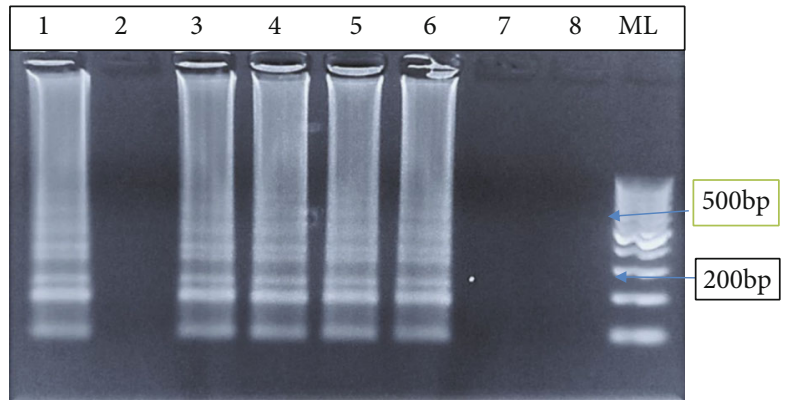

FIgURE 3: LAMP amplification results on agarose gel. Lane 1: positive control of $W$. bancrofti DNA; lane 2: negative control; lanes 3, 4, 5, and 6: positive specimens; and lanes 7 and 8: negative results. Lane $\mathrm{ML}$ is the molecular weight marker of $100 \mathrm{bp}$.

parum, Trichuris trichiura, Echinococcus granulosus, and mosquito vectors Anopheles gambiae [12, 14, 23, 27-29, 32, 38]. These parasites had been tested by PCR using specific primers for each parasite target region; their sequences are found in the gene bank.

2.7. Analytical LAMP Sensitivity Testing. To determine the sensitivity of the LAMP assay by establishing the lower detection limit, the genomic DNA concentration was determined using a NanoDrop 1000 and a 10-fold serial dilution of the DNA done. The successive serially diluted DNA was then amplified with LAMP primers to determine assay sensitivity. Also, to compare the sensitivity with the PCR sensitivity, the same dilutes were run by PCR.

2.8. Clinical LAMP Sensitivity and Specificity Testing. Clinical testing of the assay sensitivity and specificity was done; 125 clinical specimens from participants were tested on both PCR and LAMP assay. The LAMP assay sensitivity, specificity, and Kappa statistics values were determined by the $2 \times 2$ contingency table.

\section{Study Results}

The results described here show the amplification of W. bancrofti DNA using LAMP primers targeting the Ssp 1 repeat sequence. LAMP primers used in this study consistently amplified the Ssp 1 repeat sequence in a ladder like having 


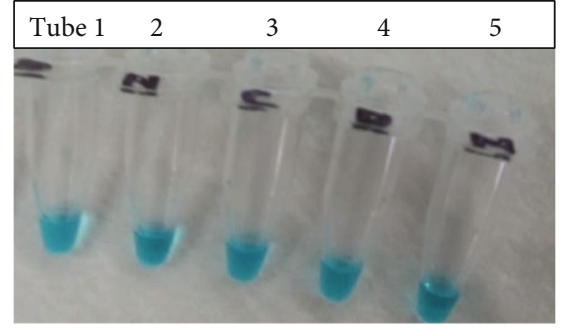

(a)

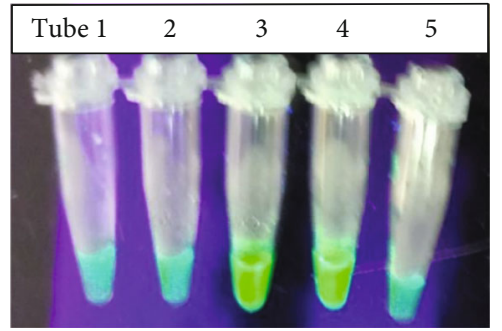

(b)

FIGURE 4: (a) LAMP reaction mix before amplification. (b) LAMP mix reaction after amplification. (a) All reaction mix in tubes 1-5 was blue in color before incubation. On incubating the reaction mix at $63^{\circ} \mathrm{C}$ for 60 minutes, the results were as shown in (b) on the addition of $1: 10 \mathrm{SYBR}$ Green dye and viewed under a UV Light or under sunlight. Tubes 1 and 2 remained blue in color indicating the absence of Wuchereria bancrofti DNA (negative specimen), tube 3 turned green in color indicating positive $W$. bancrofti DNA amplification (positive specimen), tube 4 contained $W$. bancrofti DNA-positive control which turned green in color, and tube 5 was a negative control which remained blue in color.

multiple bands of different sizes under isothermal amplification conditions of $63^{\circ} \mathrm{C}$ for 60 minutes. The test results indicated that the developed LAMP assay could successfully amplify $W$. bancrofti in a patient's blood specimen. The amplified products were detected either by use of $1: 10$ SYBR Green 1 dye by observing the color change or by agarose gel electrophoresis. A closed tube with lyophilized reaction buffers was also used to access their use in minimizing contamination and the ease of field applicability.

3.1. LAMP Amplicon Detection by Florescent Dye. Before the incubation, the color of the reaction mix was orange. In the presence of a positive LAMP DNA amplicon, the color in the reaction tube changed from orange to green and remained orange in the absence of amplicons (Figure 2).

3.2. Gel Representation of the LAMP Amplicon Results. LAMP-positive results are indicated by a stream of ladderlike light bands at different lengths, indicating the presence of $W$. bancrofti DNA in the specimen. There are no observable bands in the absence of an amplicon as shown in Figure 3.

3.3. LAMP Reaction in Closed Tubes: "Ready-to-Use Buffers." The use of closed tubes containing ready-to-use reaction buffers showed color change from original blue before amplification as shown in Figure 4(a) to light-green in the presence of $W$. bancrofti DNA in Figure 4(b) on amplification. Tubes 3 $\& 4$ on amplification at $63^{\circ} \mathrm{C}$ for one hour turned green while tubes 1,2 , \& 5 remained blue indicating the absence of $W$. bancrofti DNA as viewed under a UV light. The green color indicates a positive specimen while a negative specimen remains blue even after amplification.

3.3.1. Analytical LAMP Sensitivity and Specificity Evaluation. Our LAMP assay showed a sensitivity limit of $10^{-6}$ which was equivalent to $(1 / 1000000)$ DNA copies of the parasites in the diluent, while no band was observed in $1 / 10000000\left(1 / 10^{7}\right)$ copies in the diluent as shown in Figure 5. The assay showed a great specificity because it could only amplify $W$. bancroft $i$ DNA when tested alongside other parasites. W. bancrofti DNA showed a stream of bands at different sizes, and the absence of $W$. bancrofti DNA showed no bands (Figure 6).

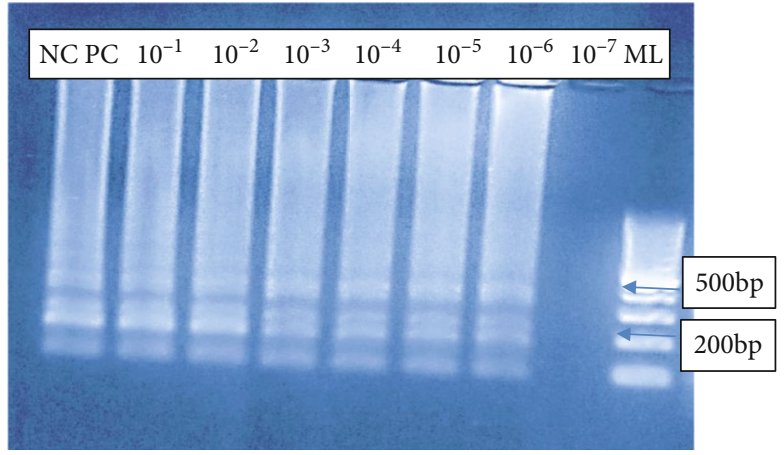

FIgURE 5: LAMP sensitivity testing on gel electrophoresis. Lane NC represents negative control; lane PC represents positive control; lanes $10^{-1}-10^{-6}$ represented serial dilutions of DNA extracts for testing the assay sensitivity. $10^{-1}-10^{-6}$ showed amplification of $W$. bancrofti as bands appeared at different lengths indicating that our LAMP assay was sensitive and could detect to up to $1 / 1000000$ $\left(1 / 10^{6}\right)$ DNA copies of the parasites in the diluent, while no band was observed in $1 / 10000000\left(1 / 10^{7}\right)$ copies in the diluent.

3.3.2. PCR Assay Sensitivity and Specificity Testing. When the same diluent used for LAMP sensitivity testing was used to run PCR, it was found that PCR could detect up to $10^{-7}$ indicating that it was a little bit more sensitive as compared to LAMP (Figure 7). There were no bands on DNA from nonW. bancrofti species.

3.4. Time-Dependent Specificity Testing Using Florescence Dye Detection. The duration of amplification time was evaluated to find out whether it has any impact on amplification; the results obtained within 30 to 60 minutes of incubation were relatively the same. There was no much color change even with the increased time for amplification at the same temperature as shown in Table 2.

3.5. Statistical Analysis of LAMP Results with PCR (Gold Standard) Results. Clinical sensitivity and specificity of LAMP was done by examining 125 patient's blood. Out of 125 samples examined, 13 were positive by PCR and 15 samples were positive by LAMP. A total of 112 samples were confirmed to be negative with PCR whereas LAMP confirmed a total of 110 specimens as negative. With respect to sensitivity, 


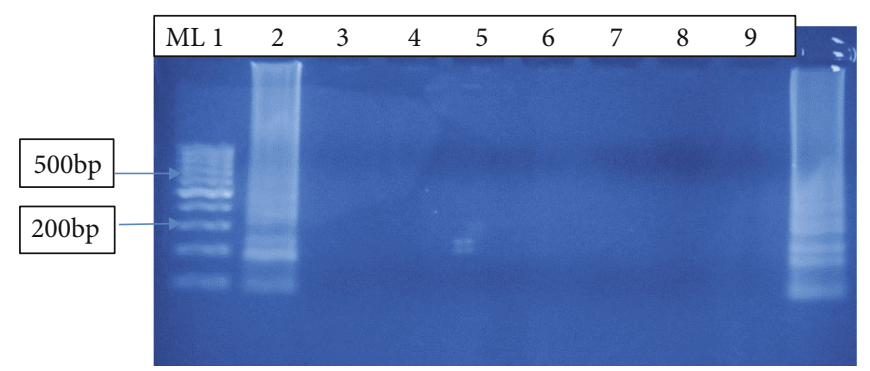

FIgURE 6: LAMP specificity testing on agarose gel electrophoresis. Lane ML is a molecular weight marker $100 \mathrm{bp}$; lane 1: W. bancrofti DNApositive specimen; lanes 2-7: non- $W$. bancrofti DNA of other parasites which did not show any amplification; lane 2: Brugia Malayi; lane 3: Anopheles gambiae; lane 4: Schistosoma mansion; lane 5: Plasmodium falciparum; lane 6: Trichuris trachura; lane 7: Echinococcosis granulosus; lane 8: negative control; lane 9: W. bancrofti DNA-positive control.

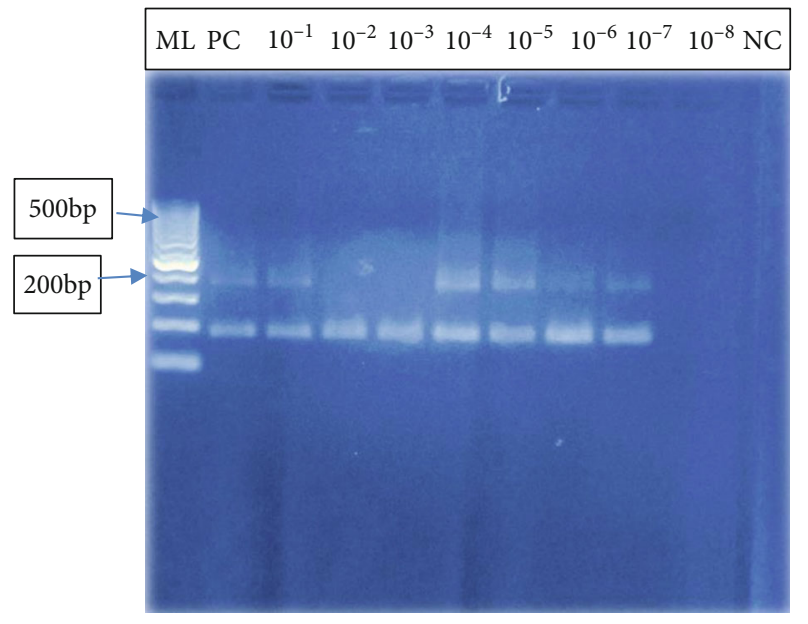

FIGURE 7: PCR sensitivity testing on gel electrophoresis. Lane ML: molecular marker $100 \mathrm{bp}$; lane PC: positive control, W. bancrofti DNA; lanes $10^{-1}-10^{-7}$ : serial dilutions of DNA extracts for testing the assay sensitivity; lane $10^{-8}$ : no amplification; lane NC: represents negative control; $10^{-1}-10^{-7}$ showed amplification of $W$. bancrofti as bands appeared just below the $200 \mathrm{bp}$ as the expected band size was $188 \mathrm{bp}$. This showed that PCR assay was sensitive and could detect to up to $1 / 1000000\left(1 / 10^{7}\right)$ copies of parasite DNA in the diluent, while no band was observed in $1 / 10000000$ $\left(1 / 10^{8}\right)$ copies in the diluent.

LAMP had a sensitivity of $92.3 \%$ and a specificity of $97.3 \%$ at $95 \%$ confidence interval and a power of 1 . When Cohen's Kappa coefficient was determined, the value was 0.84 , suggesting that there was an exceptional agreement between the two techniques.

\section{Discussion}

Accurate, specific, sensitive, affordable, and less-sophisticated diagnostic tools are needed for point-of-care detection and prompt initiation of treatment and prevention of Wuchereria bancrofti. The intensified control programs have significantly led to low human infection and low transmission rates in vectors. This has led to the need for accurate and specific tools for identifying endemic areas where treatment is required and when the transmission limit level is attained in order to declare an endemic region free of filariasis.
LAMP is a novel DNA amplification method that allows reactions to occur under isothermal conditions unlike PCR which involves cycles of varying temperatures by a specialized equipment [36]. LAMP has been developed and optimized for viruses and a number of parasites [30, 34-42]. However, studies for its application in the field are scanty. Advantages of LAMP reactions are that the use of the four to six primers-FIP, BIP, F3, B3, and/or 2 loop primers-to recognize six to eight different regions of interest in a sequence makes LAMP to have a higher specificity to the reaction compared to conventional PCR methods [36]. Another advantage using LAMP is based on the fact that the amplification from stem-loop structures leads to accumulation of large amounts of products of varying lengths. Ultimately, this makes detection of amplified DNA much easier by visual observations eliminating the need for postamplification detection by gel electrophoresis.

In this study, we evaluated the performance of LAMP assays in detection of Wuchereria bancrofti. Optimization was done by changing the primer concentrations, varying in time and temperature. The use of lyophilized reagents in a closed tube was also assessed. Detection was done by visualization through color change by use of florescence dye, 1:10 SYBR Green 1 and by gel electrophoresis. The color change, from orange to green, in the presence of amplified DNA showed an easy way of detecting amplicons (Figure 2) without the need for postamplification analysis by gel electrophoresis that uses ethidium bromide which is carcinogenic in nature, and minimizing its use is an important aspect of public health safety. The gel representation of the LAMP amplification results is as in Figure 3. These results were in accordance with the reports by Notomi et al. [29]. The closed tube in Figure 4(a) shows the reaction mix which is blue in color before amplification. After amplification at $63^{\circ} \mathrm{C}$ for 60 minutes, the results were as in Figure 4(b). In the presence of $W$. bancrofti DNA, the color changed from blue to greenish as viewed under ultraviolet light $(260 \mathrm{~nm})$ and remained blue in the absence of $W$. bancrofti DNA. The closed tubes had an added advantage since there was minimal handling of reaction reagents and this reduced the chances of cross-contamination. This study yielded results which were comparable to studies on the LAMP colorimetric test by Poon et al. [38], Goto et al. [51], and Poole et al. [52]. Tenfold serial dilution was done to test the LAMP sensitivity 
TABLE 2: Time-dependent testing.

\begin{tabular}{lccc}
\hline Sample & 30-minute amplification & $\begin{array}{c}\text { Incubation time (minutes) } \\
\text { 40-minute amplification }\end{array}$ & 60-minute amplification \\
\hline W. bancrofti-positive control & Green color & Green color & Green color \\
W. bancrofti-positive specimen (K19) & Light green color & Green color & Green color \\
S. mansoni & Orange color & Orange color & Orange color \\
Negative control & Orange color & Orange color & Orange color \\
Master Mix -Blank & Orange color & Orange color & Orange color \\
\hline
\end{tabular}

The light-green/green color indicated the presence of W. bancrofti DNA while the orange color indicates that no amplification took place.

as showed in Figures 5 and 7 for LAMP and PCR, respectively. The initial concentration was $50.0 \mathrm{ng} / \mu \mathrm{l}$ which is equivalent to $200 \mathrm{pg}$; approximately 1 (one) DNA copy was used to make the serial diluent. The clinical sensitivity of the developed LAMP was $92.3 \%$ at $95 \%$ confidence interval and with a power of 1 , with 3 more specimens testing positive as compared to PCR results. Specificity of the evaluated W. bancrofti LAMP assays was $97.3 \%$. Only specimen containing Wuchereria bancrofti species showed amplification either by color change or by gel electrophoresis as in Figure 6. Cohen's Kappa statistics showed a great agreement of 0.84 indicating that the two methods are comparable and that LAMP assays can substitute PCR in resource-limiting regions.

The $W$. bancrofti LAMP test described here shows a greater potential for use in the poorly equipped laboratories and in field setup characteristic of regions of neglected tropical disease. The detection limit for our LAMP assay was $1 / 10^{6}$ which is equivalent to 1 microfilariae per $200 \mu \mathrm{l}$ of blood (Figure 5); this gives it an advantage for use in low-prevalence and low-transmission zones.

The major challenges faced during the optimization of our $W$. bancrofti LAMP assays were the need to deal with the high rate of nonspecific amplifications that can lead to a lot of false positives. When using the opened-tube LAMP technique, there was possible cross-contamination when the lids of the reaction tubes were opened at the end of the reaction for gel electrophoresis and when adding dye for result visualization. These drawbacks faced here were similar to those reported in $[36,53-56]$. To reduce false positives and false negative, DMSO at $7.5 \%$ was used in a reaction mix and this greatly improved the LAMP specificity. It was also noted that use of the closed-tube LAMP assays minimized contamination problems because of the preprepared reaction buffers in the tubes. In addition, running gel electrophoresis at the end of the LAMP reaction requires this step to be conducted in the lab which is time-consuming and therefore not suitable for on-site rapid detection.

\section{Conclusion}

We have optimized LAMP assays capable of detecting Wuchereria bancrofti in human blood. The assays are highly sensitive and species specific and can be used with a wide variety of DNA templates (genomic DNA, extracted DNA, boiled fresh whole blood, or blood spot samples). When closed tubes (lyophilized reagents) were applied to clinical samples, the LAMP assays were very promising and represented a powerful alternative to PCR. The LAMP method will be of benefit to global health programs aimed at eliminating filarial infections.

\section{Recommendations}

We recommend the use of closed-tube reaction with premix to avoid cross-contamination which was a major challenge throughout our study during the open-tube analysis. Proper primer designing and assay optimization are required to avoid false positives as a result of primer dimers.

\section{Data Availability}

All data is included in the text.

\section{Conflicts of Interest}

The author(s) declare(s) that they have no conflicts of interest.

\section{Authors' Contributions}

NMK conceived the study, developed the proposal, sourced the funding, coordinated and implemented all the aspects of the study, and developed the manuscript. WD, IC, and WE developed the proposal, analyzed the data, and drafted the manuscript. LJ and GR were involved in organizing and carrying out the field and laboratory activities and drafting of the manuscript. WL, MW, LK, and MC helped in the developing the proposal and guiding and supervising the laboratory assays and review of the manuscript. KJ was involved in proposal development, giving guidance and supervision of the study, and more importantly, providing the LAMP assays kits for this study.

\section{Acknowledgments}

This project was funded by the National Council of Science, Technology and Innovation (NACOSTI), (NRF) National Research Fund, Kenya, ref. NACOSTI/RCD/ST \& I $6^{\text {th }}$ call $\mathrm{PhD} / 156$. The authors wish to thank the Kenya Medical Research Institute and the Filariasis Research Unit where the research was carried out and for authorizing the publication of this work. Special thanks goes to Kimani Francis of CBRD for their kind guidance throughout the study. 


\section{References}

[1] WHO, "Global programme to eliminate lymphatic filariasis: progress report, 2014," Weekly Epidemiological Record= Relevé épidémiologique hebdomadaire, vol. 90, pp. 489-504, 2015.

[2] M. J. Bockarie, M. J. Taylor, and J. O. Gyapong, "Current practices in the management of lymphatic filariasis," Expert review of anti-infective therapy, vol. 7, no. 5, pp. 595-605, 2009.

[3] WHO, Lymphatic Filariasis: A Handbook for National Elimination Programmes, World Health Organization, Geneva, Switzerland, 2013.

[4] S. Wynd, W. D. Melrose, D. N. Durrheim, J. Carron, and M. Gyapong, "Understanding the community impact of lymphatic filariasis: a review of the sociocultural literature," Bulletin of the World Health Organization, vol. 85, no. 6, pp. 493498, 2007.

[5] WHO, "Global programme to elimination lymphatic filariasis progress report, 2013," Weekly Epidemiological Record= Relevé épidémiologique hebdomadaire, vol. 89, no. 38, pp. 409-420, 2014.

[6] WHO, Neglected tropical diseases, 2020, June 2020, https:// www.who.int/neglected_diseases/diseases/en/.

[7] World Health Organisation, Annual Report of the Global Programme to Eliminate Lymphatic Filariasis, WHO, Geneva, 2002.

[8] A. Deshpande, M. K. Miller-Petrie, P. A. Lindstedt et al., “The global distribution of Lymphatic filariasis, 200-2018; a geospatial analysis," The Lancet Global Health, vol. 8, no. 9, pp. E1186-E1194, 2020.

[9] R. Gounoue-Kamkumo, H. C. Nana-Djeunga, J. Bopda, J. Akame, A. Tarini, and J. Kamgno, "Loss of sensitivity of immunochromatographic test (ICT) for lymphatic filariasis diagnosis in low prevalence settings: consequence in the monitoring and evaluation procedures," BMC Infectious Diseases, vol. 15, no. 1, p. 579, 2015.

[10] J. E. RosenBlatt, "Laboratory diagnosis of infections due to blood and tissue parasites," Clinical Infectious Diseases, vol. 49, no. 7, pp. 1103-1108, 2009.

[11] WHO, "Integrated vector management to control malaria and lymphatic filariasis," in WHO position statement, 2011, World Health Organization, Geneva, 2011.

[12] N. Momar, "Diagnosis of parasitic diseases: old and new approaches," Interdisciplinary Perspectives on Infectious Diseases, vol. 2009, Article ID 278246, 15 pages, 2009.

[13] G. J. Weil and R. M. Ramzy, "Diagnostic tools for filariasis elimination programs," Trends Parasitology, vol. 23, pp. 7882, 2007.

[14] K. V. Rao, M. Eswaran, V. Ravi, B. Gnanasekhar, and R. B. Narayanan, "The Wuchereria bancrofti orthologue of Brugia malayi SXP1 and the diagnosis of bancroftian filariasis," Molecular and Biochemical Parasitology, vol. 107, no. 1, pp. 71-80, 2000.

[15] E. A. Ottesen and W. C. Campbell, "Ivermectin in human medicine," Journal of Antimicrobial Chemotherapy, vol. 34, no. 2, pp. 195-203, 1994.

[16] P. J. Lammie, G. Weil, R. Noordin et al., "Recombinant antigen-based antibody assays for the diagnosis and surveillance of lymphatic filariasis - a multicenter trial," Filarial Journal, vol. 3, no. 1, p. 9, 2004.

[17] C. M. Steel, J. Kubofcik, E. A. Ottesen, and T. B. Nutman, "Antibody to the filarial antigen $\mathrm{Wb} 123$ reflects reduced trans- mission and decreased exposure in children born following single mass drug administration (MDA)," Plos Neglected Tropical Diseases, vol. 6, no. 12, article e1940, 2012.

[18] M. Zhong, J. McCarthy, L. Bierwert, M. Lizotte-Waniewski, and S. Chanteau, "A polymerase chain reaction assay for detection of the parasite Wuchereria bancrofti in human blood samples," American Journal of Tropical Medicine and Hygiene, vol. 54, no. 4, pp. 357-363, 1996.

[19] R. M. Ramzy, H. A. Farid, I. H. Kamal, G. H. Ibrahim, and Z. S. Morsy, "A polymerase chain reaction-based assay for detection of Wuchereria bancrofti in human blood and Culex pipiens," Transactions of Royal Society of Tropical Medicine and Hygiene, vol. 91, no. 2, pp. 156-160, 1997.

[20] R. M. Ramzy, "Field application of PCR-based assays for monitoring Wuchereria bancrofti infection in Africa," Annals of Tropical Medicine \& Parasitology, vol. 96, no. 2, pp. S55-S59, 2002.

[21] S. Nuchprayoon, "DNA- based diagnosis of lymphatic filariasis," Southeast Asian Journal of Tropical Medicine and Public Health, vol. 40, no. 5, pp. 904-913, 2009.

[22] P. Fischer, I. Bonow, T. Supali, P. Ruckert, and N. Rahmah, "Detection of filaria-specific IgG4 antibodies and filarial DNA, for the screening of blood spots for Brugia timori," Annals of Tropical Medicine and Parasitology, vol. 99, no. 1, pp. 53-60, 2005.

[23] M. L. Bockarie, P. Fischer, S. A. Williams, P. A. Zimmerman, and L. Griffin, "Application of a polymerase chain reactionELISA to detect Wuchereria bancrofti in pools of wildcaught Anopheles punctulatus in a filariasis control area in Papua New Guinea," American Journal of Tropical Medicine and Hygiene, vol. 62, pp. 363-367, 2000.

[24] D. L. Fink, G. A. Fahle, S. Fischer, D. F. Fedorko, and T. B. Nutman, "Toward molecular parasitologic diagnosis: enhanced diagnostic sensitivity for filarial infections in mobile populations," Journal of Clinical Microbiology, vol. 49, no. 1, pp. 4247, 2011.

[25] D. S. Goodman, J. N. Orelus, J. M. Roberts, P. J. Lammie, and T. G. Streit, "PCR and mosquito dissection as tools to monitor filarial infection levels following mass treatment," Filaria Journal, vol. 2, no. 11, 2003.

[26] S. A. Williams, S. J. Laney, L. A. Bierwert, L. J. Saunders, and D. A. Boakye, "Development and standardization of a rapid, PCR-based method for the detection of Wuchereria bancrofti in mosquitoes, for xenomonitoring the human prevalence of bancroftian filariasis," Annals of Tropical Medicine and Parasitology, vol. 96, Suppl 2, pp. S41-S46, 2002.

[27] S. J. Laney, C. J. Buttaro, S. Visconti, N. Pilotte, and R. M. Ramzy, "A reverse transcriptase-PCR assay for detecting filarial infective larvae in mosquitoes," PLoS of Neglected Tropical Diseases, vol. 2, no. 6, article e251, 2008.

[28] P. M. Intapan, T. Thanchomnang, V. Lulitanond, and W. Maleewong, "Rapid detection of Wuchereria bancrofti and Brugia malayi in mosquito vectors (Diptera: Culicidae) using a real-time fluorescence resonance energy transfer multiplex PCR and melting curve analysis," Journal of Medical Entomology, vol. 46, no. 1, pp. 158-164, 2009.

[29] T. Notomi, H. Okayama, H. Masubuchi et al., "Loop-mediated isothermal amplification of DNA," Nucleic acids research, vol. 28, no. 12, p. E63, 2000.

[30] L. L. Poon, C. S. Leung, M. Tashiro et al., "Rapid detection of the severe acute respiratory syndrome (SARS). Coronavirus 
by a loop-mediated isothermal amplification assay," Clinical Chemistry, vol. 501, pp. 50-52, 2004.

[31] C. M. Mugasa, T. Laurent, G. J. Schoone, P. A. Kager, G. W. Lubega, and H. D. F. H. Schalling, "Nucleic acid sequencebased amplification with oligochromatography (NASBA-OC) for the detection of Trypanosoma brucei in clinical samples," Journal of Clinical Microbiology, vol. 47, no. 3, p. 630, 2009.

[32] H. Takagi, M. Itoh, S. Kasai, T. C. Yahathugoda, and M. V. Weerasooriya, "Development of loop-mediated isothermal amplification method for detecting DNA in human blood and vector mosquitoes," Parasitology International, vol. 60, pp. 493-497, 2011.

[33] L. M. Zanoli and G. Spoto, "Isothermal amplification methods for the detection of nucleic acids in microfluidics.device," Biosensors, vol. 3, pp. 18-43, 2013.

[34] M. Parida, G. Posadas, S. Inoue, F. Hasebe, and K. Morita, "Real-time reverse transcription loop-mediated isothermal amplification for rapid detection of West Nile virus," Journal of Clinical Microbiology, vol. 42, no. 1, pp. 257-263, 2004.

[35] S. Y. Liang, Y. H. Chan, and K. T. Hsia, "Development of loopmediated isothermal amplification assay for detection of Entamoeba histolytica," Journal of Clinical Microbiology, vol. 47, no. 6, pp. 1892-1895, 2009.

[36] Z. K. Njiru, A. S. J. Mikosza, and E. Matovu, “African trypanosomiasis: sensitive and rapid detection of the sub-genus Trypanozoon by loop-mediated isothermal amplification (LAMP) of parasite DNA," International Journal for Parasitology, vol. 38, no. 5, pp. 589-599, 2008.

[37] A. Nkouawa, Y. Sako, M. Nakao, K. Nakaya, and A. Ito, "Loopmediated isothermal amplification method for differentiation and rapid detection of Taenia species," Journal of Clinical Microbiology, vol. 47, no. 1, pp. 168-174, 2009.

[38] L. L. Poon, B. W. Wong, E. H. Ma et al., "Sensitive and inexpensive molecular test for falciparum malaria: detecting Plasmodium falciparum DNA directly from heat-treated blood by loop-mediated isothermal amplification," Clinical Chemistry, vol. 52, no. 2, pp. 303-306, 2006.

[39] M. A. Bakheit, D. Torra, L. A. Palomino et al., "Sensitive and specific detection of Cryptosporidium species in PCRnegative samples by loop-mediated isothermal DNA amplification and confirmation of generated LAMP products by sequencing," Veterinary parasitology, vol. 158, no. 1-2, pp. 11-22, 2008.

[40] H. Iseki, A. Alhassan, N. Ohta et al., "Development of a multiplex loop-mediated isothermal amplification (mLAMP) method for the simultaneous detection of bovine Babesia parasites," Journal of Microbiology Methods, vol. 71, no. 3, pp. 281-287, 2007.

[41] G. Guan, A. Chauvin, J. Luo et al., “The development and evaluation of a loop-mediated isothermal amplification (LAMP) method for detection of Babesia spp. infective to sheep and goats in China," Experimental Parasitology, vol. 120, no. 1, pp. 39-44, 2008.

[42] H. Aonuma, M. Suzuki, H. Iseki et al., "Rapid identification of Plasmodium -carrying mosquitoes using loop-mediated isothermal amplification," Biochemical and Biophysical Research Communications, vol. 376, no. 4, pp. 671-676, 2008.

[43] Y. Mori, K. Nagamine, N. Tomita, and T. Notomi, "Detection of loop-mediated isothermal amplification reaction by turbidity derived from magnesium pyrophosphate formation," Biochemical and Biophysical Research Communications, vol. 289, no. 1, pp. 150-154, 2001.
[44] H. Aonuma, A. Yoshimura, N. Perera et al., "Loop-mediated isothermal amplification applied to filarial parasites detection in the mosquito vectors: Dirofilaria immitis as a study model," Parasites and Vectors, vol. 2, no. 1, p. 15, 2009.

[45] J. M. Kagai, S. Mpoke, F. Muli, J. Hamburger, and E. U. Kenya, "Molecular technique utilizing sputum for detecting Wuchereria bancrofti infections in Malindi, Kenya," East Africa Medical Journal, vol. 85, no. 3, pp. 28-33, 2008.

[46] S. M. Njenga, H. M. Kanyi, F. M. Mutungi et al., "Assesment of lymphatic filariasis prior to restarting mass drug administration campaigns in coastal Kenya," Parasites \& Vectors, vol. 10, no. 99, pp. 2044-2045, 2017.

[47] Districts of Kenya, 2012, http://Statoids.com/.

[48] S. Datta, S. Maitra, P. Gayen, and S. P. Sinha Babu, "Absence of symbiotic Wolbachia endobacteria in Setaria cervi from Birbhum, West Bengal, India," Current Science, vol. 93, pp. 2223, 2007.

[49] L. Nicolas and C. Plichart, "A universally applicable internal standard for PCR detection of Wuchereria bancrofti in biological samples," Parasite, vol. 4, no. 3, pp. 253-257, 1997.

[50] D. G. Wang, J. D. Brewster, M. Paul, and P. M. Tomasula, "Two methods for increased specificity and sensitivity in loop-mediated isothermal amplification," Molecules, vol. 20, no. 4, pp. 6048-6059, 2015.

[51] M. Goto, E. Honda, A. Ogura, A. Nomoto, and K. Hanaki, "Colorimetric detection of loop-mediated isothermal amplification reaction by using hydroxy naphthol blue," BioTechniques, vol. 46, no. 3, pp. 167-172, 2009.

[52] C. B. Poole, Z. Li, A. Alhassan et al., "Colorimetric tests for diagnosis of filarial infection and vector surveillance using non-instrumented nucleic acid loop-mediated isothermal amplification (NINALAMP)," PLoS ONE, vol. 12, no. 2, 2017.

[53] D. C. Nyan, L. E. Ulitzky, N. Cehan et al., "Rapid detection of hepatitis B virus in blood plasma by a specific and sensitive loop-mediated isothermal amplification assay," Clinical infectious diseases, vol. 59, pp. 16-23, 2014.

[54] M. Parida, S. Sannarangaiah, P. K. Dash, P. V. Rao, and K. Morita, "Loop mediated isothermal amplification (LAMP): a new generation of innovative gene amplification technique; perspectives in clinical diagnosis of infectious diseases," Reviews in medical virology, vol. 18, pp. 407-421, 2008.

[55] Y. L. Lau, P. Meganathan, P. Sonaimuthu, G. Thiruvengadam, V. Nissapatorn, and Y. Chen, "Specific, sensitive and rapid diagnosis of active toxoplasmosis by a loop-mediated isothermal amplification method using blood samples from patients," Journal of Clinical Microbiology, vol. 48, no. 10, pp. 3698-3702, 2010.

[56] R. Angamuthu, S. Baskaran, D. R. Gopal, J. Deverajan, and K. Kathaperumal, "Rapid detection of the Marek's disease viral genome in chicken feathers by loop-mediated isothermal amplification," Journal of clinical microbiology, vol. 50, pp. 961-965, 2011. 